PROCEEDINGS OF THE

AMERICAN MATHEMATICAL SOCIETY

Volume 130, Number 3, Pages 631-635

S 0002-9939(01)06116-0

Article electronically published on August 28, 2001

\title{
VAN DER WAERDEN SPACES
}

\author{
MENACHEM KOJMAN \\ (Communicated by Alan Dow)
}

\begin{abstract}
A topological space $X$ is van der Waerden if for every sequence $\left(x_{n}\right)_{n}$ in $X$ there exists a converging subsequence $\left(x_{n_{k}}\right)_{k}$ so that $\left\{n_{k}: k \in \mathbb{N}\right\}$ contains arbitrarily long finite arithmetic progressions. Not every sequentially compact space is van der Waerden. The product of two van der Waerden spaces is van der Waerden.

The following condition on a Hausdorff space $X$ is sufficent for $X$ to be van der Waerden:

(*) The closure of every countable set in $X$ is compact and first-countable.

A Hausdorff space $X$ that satisfies $(*)$ satisfies, in fact, a stronger property: for every sequence $\left(x_{n}\right)$ in $X$ :

( $\star$ ) There exists $A \subseteq \mathbb{N}$ so that $\left(x_{n}\right)_{n \in A}$ is converging, and $A$ contains arbitrarily long finite arithmetic progressions and sets of the form $F S(D)$ for arbitrarily large finite sets $D$.

There are nonmetrizable and noncompact spaces which satisfy $(*)$. In particular, every sequence of ordinal numbers and every bounded sequence of real monotone functions on $[0,1]$ satisfy $(\star)$.
\end{abstract}

\section{INTRODUCTION}

Let us recall van der Waerden's Theorem. Let $\mathbb{N}=\{0,1, \ldots\}$ denote the set of natural numbers.

Definition 1. A set $A$ of natural numbers is called an AP set if it contains arbitrarily long finite arithmetic progressions.

Theorem 2 (van der Waerden [9]). If $\mathbb{N}$ is partitioned to finitely many parts, then one of the parts is an AP set.

An equivalent formulation of van der Waerden's theorem is that every sequence in a finite (Hausdorff) topological space has a converging subsequence whose set of indices contains arbitrarily long finite arithmetic progressions. In this paper we address the problem of which topological spaces satisfy this topological formulation of van der Waerden's Theorems. The following condition $(*)$ on a space $X$ will be

Received by the editors August 2, 2000 and, in revised form, August 28, 2000.

2000 Mathematics Subject Classification. Primary 05C55, 54F65; Secondary 03E05, 11P99, $26 \mathrm{~A} 48$.

Key words and phrases. van der Waerden's Theorem, converging sequence, compactification, finite sums.

The author thanks Uri Abraham for many discussions that contributed to the development of this paper, and also thanks the referee for some constructive and helpful comments.

(C)2001 American Mathematical Society 
a sufficient condition in two different generalized van der Waerden type theorems (10) and 11 below):

(*) The closure of every countable set in $X$ is compact and first-countable.

\section{VAN DER WAERDEN SPACES}

Definition 3. (1) A topological space $X$ is van der Waerden if for every sequence $\left(x_{n}\right)_{n}$ in $X$ there exists a converging subsequence $\left(x_{n_{k}}\right)_{k}$ so that $\left(n_{k}\right)_{k}$ is AP.

(2) A topological space $X$ is strongly van der Waerden if for every AP set $A \subseteq \mathbb{N}$ and a sequence $\left(x_{n}\right)_{n \in A}$ in $X$ there exists a converging subsequence $\left(x_{n}\right)_{n \in B}$ where $B \subseteq A$ is $\mathrm{AP}$

Proposition 4. A space $X$ is van der Waerden if and only if it is strongly van der Waerden.

Proof. Suppose $A \subseteq \mathbb{N}$ is $\mathrm{AP}$ and $f: A \rightarrow X$ is given. By thinning $A$ out we may assume that $A=\bigcup C_{n}$ where each block $C_{n}$ is an arithmetic progression, $\sup C_{n}<\min C_{n+1}$ and $\left|C_{n+1}\right|>\left(\max C_{n}\right)^{2}$.

Let $h: \mathbb{N} \rightarrow A$ be the increasing enumeration of $A$. Let $g: \mathbb{N} \rightarrow X$ be defined by $g(n)=f(h(n))$ for all $n \in \mathbb{N}$. Since $X$ is van der Waerden, there exists an AP set $B \subseteq \mathbb{N}$ so that $g\lceil B=(f \circ h)\lceil B$ is converging. We claim that $h[B]$ is also AP, hence $f\lceil h[B]$ is as required.

To see that $h[B]$ is AP, let $k$ be arbitrary. Let $C=\left\{a_{0}, a_{0}+d, \ldots, a_{0}+(k-1) d\right\} \subseteq$ $B$ be an arithmetic progression of length $k$ so that $k<a_{0}$. Observe that for each block $C_{n}$ of $A, h[C] \cap C_{n}$ is an arithmetic progression.

Let $C_{n_{0}}$ be the block in $A$ to which $h\left(a_{0}+d\right)$ belongs. Clearly $k, a_{0}$ and $d$ are smaller than $\max C_{n_{0}}$, hence $a_{0}+(k-1) d \leq \max C_{n_{0}}+\left(\max C_{n_{0}}-1\right)\left(\max C_{n_{0}}\right)=$ $\left(\max C_{n_{0}}\right)^{2}<\left|C_{n_{0}+1}\right|$. Since $h$ is the increasing enumeration of $\bigcup C_{n}$, it holds that $h\left(a_{0}+(k-1) d\right) \in C_{n_{0}} \cup C_{n+1}$, and consequently $h[C] \subseteq C_{n_{0}} \cup C_{n_{0}+1}$. Thus either $h[C] \cap C_{n_{0}}$ or $h[C] \cap C_{n_{0}+1}$ is an arithmetic progression of length $\geq(k-1) / 2$.

Corollary 5. The product of two van der Waerden spaces is van der Waerden.

Proof. Suppose $Z=X \times Y$ where $X, Y$ are van der Waerden and suppose $f: \mathbb{N} \rightarrow Z$ is given. Put $f(n)=\left(x_{n}, y_{n}\right)$. Since $X$ is van der Waerden, there is an AP set $A \subseteq \mathbb{N}$ so that $\left(x_{n}\right)_{n \in A}$ is converging. Since $Y$ is strongly van der Waerden, there is an AP set $B \subseteq A$ so that $\left(y_{n}\right)_{n \in B}$ is converging. Clearly, $(f(n))_{n \in B}$ is converging in $Z$.

Every van der Waerden space is surely sequentially compact. The next example shows that being van der Waerden is strictly stronger than being sequentially compact.

Theorem 6. There exists a Hausdorff, compact, sequentially compact, separable space which is first-countable at all points but one, which is not van der Waerden.

Proof. Let $\mathcal{F}$ be a maximal member of $\{\mathcal{A}: \mathcal{A}$ is an infinite set of almost disjoint infinite subsets of $\mathbb{N}$ and each $A \in \mathcal{A}$ is not AP $\}$. Then in fact, $\mathcal{F}$ is a maximal almost disjoint family. (If $\mathcal{F}$ is not maximal as an almost disjoint family, fix some infinite $B \subseteq \mathbb{N}$ which has finite intersection with every $A \in \mathcal{F}$. By thinning out, $B$ can be made non-AP.)

For every $A \in \mathcal{F}$, let $p_{A} \notin \mathbb{N}$ be a distinct point. Let $Y=\mathbb{N} \cup\left\{p_{A}: A \in \mathcal{F}\right\}$ and let $\mathcal{T}=\left\{B \subseteq Y\right.$ : for all $A \in \mathcal{F}$, if $p_{A} \in B$, then $A \backslash B$ is finite $\}$. Then with the 
topology $\mathcal{T}, Y$ is a locally compact Hausdorff space with $\mathbb{N}$ as a discrete subspace. Let $X=Y \cup\{p\}$ be the one point compactification of $Y$.

$X$ is compact, separable, and at every point except $p, X$ is first countable. To check that $X$ is sequentially compact, suppose that $\left(x_{n}\right)$ is a sequence in $X$. If $\left\{x_{n}: n \in \mathbb{N}\right\}$ is finite, then $\left(x_{n}\right)_{n}$ has a constant subsequence. Otherwise, we may assume, without loss of generality, that $\left(x_{n}\right)_{n}$ is one-to-one and that either $\left\{x_{n}: n \in \mathbb{N}\right\} \subseteq \mathbb{N}$ or $\left\{x_{n}: n \in \mathbb{N}\right\} \subseteq\left\{p_{A}: A \in \mathcal{F}\right\}$. In the first case, maximality of $\mathcal{F}$ implies the existence of some $A \in \mathcal{F}$ and some subsequence $\left(x_{n_{k}}\right)_{k}$ so that $x_{n_{k}} \in A$ for all $k$; this subsequence converges to $p_{A}$. In the second case, $\left(x_{n}\right)_{n}$ itself converges to $p$. Thus $X$ is sequentially compact.

To see that $X$ is not van der Waerden, let $x_{n}=n$. Every convergent subsequence $\left(x_{n_{k}}\right)_{k}$ satisfies that for some $A \in \mathcal{F},\left\{n_{k}: k \in \mathbb{N}\right\} \backslash A$ is finite. By the choice of $\mathcal{F}$, $\left\{n_{k}: k \in \mathbb{N}\right\}$ is not AP.

The following is a well-known, equivalent, finite version of van der Waerden's theorem:

Theorem 7. For all $k$ and $\ell$ there exists $n$ such that for every partition of $n=$ $\{0,1, \ldots, n-1\}$ to $\ell$ parts, one of the parts contains an arithmetic progression of length $k$.

One immediately sees that the finite version implies the following equivalent formulation of van der Waerden's theorem:

Theorem 8. For every partition of an AP set to finitely many parts, one of the parts is $A P$.

This formulation also follows directly from Proposition 4 applied to finite spaces.

Corollary 9. The collection of all complements of non-AP sets is a uniform filter $\mathcal{A}$ over $\mathbb{N}$ which consists of AP sets. An ultrafilter $U$ satisfies that every member of $U$ is $A P$ if and only if $\mathcal{A} \subseteq U$.

Theorem 10. Suppose a Hausdorff space $X$ satisfies the following condition:

(*) The closure of every countable set in $X$ is compact and first-countable.

Then $X$ is van der Waerden.

Proof. Suppose that $\left(x_{n}\right)_{n \in \mathbb{N}} \subseteq X$ is given. Let $D=\operatorname{cl}_{X}\left\{x_{n}: n \in \mathbb{N}\right\}$. Let $U$ be an ultrafilter over $\mathbb{N}$ which extends $\mathcal{A}$. Thus every set $A \in U$ is AP. Define an ultrafilter $U^{\prime}$ over $D$ by $A \in U^{\prime} \Longleftrightarrow\left\{n: x_{n} \in A\right\} \in U$. By compactness (and Hausdorffness) of $D$, there is a unique point $x \in D$ with the property that every neighborhood (in $D$ ) of $x$ belongs to $U^{\prime}$. Fix, by first-countability, a decreasing neighborhood base $\left(u_{k}\right)_{k}$ at $x$. For every $k$, since $\left\{n: x_{n} \in u_{k}\right\} \in U$, the set $\left\{n: x_{n} \in u_{k}\right\}$ contains arbitrarily long arithmetic progressions. For each $k$, choose an arithmetic progression $C_{k} \subseteq \mathbb{N}$ of length $k$ so that $\left\{x_{n}: n \in C_{k}\right\} \subseteq u_{k}$. The set $B=\bigcup_{k} C_{k}$ is AP and $\left(x_{n}\right)_{n \in B}$ converges to $x$.

2.1. A strengthening. For a nonempty set $D \subseteq \mathbb{N}$ denote by $F S(D)$ the set of all finite sums over $D$. A finite sum is an integer of the form $\sum_{n \in \alpha} n$ where $\alpha \subseteq D$ is finite and nonempty. Call a subset $A \subseteq \mathbb{N}$ an IP set if there exists an infinite set $D \subseteq \mathbb{N}$ so that $F S(D) \subseteq A$. 
Theorem 10 can be considerably strengthened using the technology of idempotents in $(\beta \mathbb{N},+) . \beta \mathbb{N}$ is the set of all ultrafilters over $\mathbb{N}$ and the operation of addition in $\mathbb{N}$ can be extended to $\beta \mathbb{N}$ via the formula

$$
A \in U+V \Longleftrightarrow\{n: A-n \in U\} \in V .
$$

For a good exposition of this material see 2] (with the caution that they reverse the operation from the way it is described in (11)). An ultrafilter $U \in \beta \mathbb{N}$ is an idempotent if $U$ is nonprincipal and $U+U=U$, and an idempotent $U$ is a minimal idempotent if it belongs to a minimal right ideal in $(\beta \mathbb{N},+)$. If $U$ is an idempotent, then every set $A \in U$ is IP, and if $U$ is a minimal idempotent, then every set in $U$ is AP (Theorems 4.40 and 14.1 in [2]).

Theorem 11. Suppose a Hausdorff space $X$ satisfies condition (*). Then for every sequence $\left(x_{n}\right)_{n \in \mathbb{N}} \subseteq X$ there exists a set $A \subseteq \mathbb{N}$ so that

(1) $\left(x_{n}\right)_{n \in A}$ is converging;

(2) A contains arbitrarily long arithmetic progressions;

(3) A contains $F S(D)$ for arbitrarily large finite sets $D$.

Proof. Let $U$ be a minimal idempotent in $(\beta \mathbb{N},+)$. Suppose $\left(x_{n}\right)_{n \in N}$ is given, and let $U^{\prime}$ be the corresponding ultrafilter on $\operatorname{cl}_{X}\left\{x_{n}: n \in \mathbb{N}\right\}$. Let $x \in \operatorname{cl}_{X}\left\{x_{n}: n \in \mathbb{N}\right\}$ satisfy that every neighborhood of $x$ belongs to $U^{\prime}$, and let $u_{n}$ be a decreasing neighborhood base at $x$. Since every set in $U$ contains both arbitrarily long arithmetic progressions and an IP set, one can fix $F S\left(D_{n}\right)$ and an arithmetic progression $C_{n}$ inside $\left\{k: x_{k} \in u_{n}\right\}$ with $\left|C_{n}\right|,\left|D_{n}\right| \geq n$. Now $\bigcup_{n} C_{n} \cup F S\left(D_{n}\right)$ is as required.

\section{COnCluding Remarks}

The class of spaces which satisfy $(*)$ includes all compact metric spaces, all compact linearly ordered topological spaces and every limit ordinal of uncountable cofinality. In particular,

Corollary 12. Every sequence $\left(\alpha_{n}\right)$ of ordinal numbers contains a converging subsequence $\left(\alpha_{n_{k}}\right)$ so that $\left\{n_{k}: k \in \mathbb{N}\right\}$ contains arbitrarily long arithmetic progressions and $F S(D)$ for arbitrarily large finite sets $D$.

The space $\omega_{2}$ with the order topology is an example of a space that satisfies $(*)$ which is neither first-countable nor compact (but all of its separable subspaces are metrizable). Helly's space of all monotone functions from $[0,1]$ to $[0,1]$ with the topology induced by pointwise convergence (see [3]) is an example of a separable, compact and first-countable space - which, therefore, satisfies $(*)$ - which is not metrizable. The instance of Theorem 11 for this particular space gives:

Corollary 13. Suppose that $f_{n}:[0,1] \rightarrow[0,1]$ is monotone for each $n$. Then there is pointwise converging subsequence $\left(f_{n_{k}}\right)$ so that $\left\{n_{k}: k \in \mathbb{N}\right\}$ is AP and contains $F S(D)$ for arbitrarily large finite sets $D$.

\section{REFERENCES}

[1] Ronald L. Graham, Bruce L. Rothschild and Joel H. Spencer. Ramsey Theory, John Wiley E Sons, 1990. MR 90m:05003

[2] N. Hindman and D. Strauss. Algebra in the Stone-Čech compactification, de Gruyter Berlin, 1998. MR 99j:54001

[3] Lynn Arthur Steen and J. Arthur Seebach Jr., Counterexamples in topology, Springer-Verlag, New York, 1978. MR 80a:54001 
[4] V. Bergelson. Ergodic Ramsey Theory - an update. Ergodic theory and $Z^{d}$-actions. London Math. Soc. Lecture Note series 228, 1-61, 1996. MR 98g:28017

[5] V. Bergelson and N. Hindman. Nonmetrizable topological dynamics and Ramsey Theory. Trans. Amer. Math. Soc., 320:293-320, 1990. MR 90k:03046

[6] N. Hindman. Finite sums from sequences within cells of partitions of N. J. Combinatorial Theory Ser. A, 17:119-184, 1974. MR 50:2067

[7] N. Hindman. Ultrafilters and combinatorial number theory. Number Theory, Carbondale, M. Nathanson, ed. Lecture Notes in Math., 751, 1979. MR 81m:10019

[8] N. Hindman. Partitions and sums and products of integers. Trans. Amer. Math. Soc., 247:227245, 1979. MR 80b: 10022

[9] B. L. van der Waerden. Beweis eine Baudetschen Vermutung Nieus Arch. Wisk., 15:212-216, 1927.

Department of Mathematics, Ben Gurion University of the Negev, Beer Sheva 84105, ISRAEL

E-mail address: kojman@math.bgu.ac.il 